\title{
HISTÓRICOS CURSOS DE PSICOLOGIA SOCIAL NO BRASIL
}

Elizabeth de Melo Bomfim

\author{
Universidade Federal de São João Del-Rei
}

RESUMO:O artigo ancora-se numa reflexão sobre quatro cursos de Psicologia Social ministrados no Brasil na primeira metade do século XX. O legado documental de seus proponentes traz à cena vestígios das práticas educativas, informando o teor temático e metodológico assim como as influências teóricas. Aberta às contribuições de vários campos de conhecimento, a Psicologia Social é revelada em seu momento de imprecisão, fragilidade e construção como campo científico.

PALAVRAS-CHAVE: Ensino de Psicologia Social - História da Psicologia Social - Construção do Conhecimento Científico.

\section{HISTORICAL COURSES OF SOCIAL PSYCHOLOGY IN BRAZIL}

ABSTRACT: The article is a reflection on four courses of Social Psychology ministered in Brazil during the first half of the twentieth century. The documental legacy brings to light educational practices, informing us of its thematical and methodological contents besides theoretical influences. Opened to the contributions of different fields of knowledge, Social Psychology shows itself in a moment of fragility, imprecision and construction as a scientific field.

KEY-WORDS: Social Psychology Education - Social Psychology History - Construction of Scientific Knowledge

\section{INTRODUÇÃO}

O percurso do ensino de Psicologia Social no Brasil é, até hoje, pouco conhecido. A escassez de documentos escritos e de registros audiovisuais aliada à raridade de pesquisas em relação à temática abre lacunas na compreensão das práticas educativas em Psicologia Social. Os parcos registros documentais existentes em relação à primeira metade do século XX, no Brasil, só permitem trazer à cena vestígios dessas práticas, restringindo as informações ao teor temático e metodológico e às influências teóricas. O que se propõe nesse momento é articular os desdobramentos desses registros existentes que - quer pelo pioneirismo, quer pela relevância - marcaram o trajeto do ensino de Psicologia Social no país.

Elegem-se, assim, quatro cursos de Psicologia Social que, em suas diversidades, apontaram o momento de imprecisão, fragilidade e construção do campo científico. São eles: o curso ministrado por Raul Carlos Briquet, no segundo semestre de 1933, na Escola Livre de Sociologia e Política de São Paulo; o curso ministrado por Arthur Ramos, em 1935, na Escola de Economia e Direito da extinta Universidade do Distrito Federal situada no Rio de Janeiro; o curso ministrado por Donald Pierson, na década de 1940, na Escola Livre de Sociologia e Política de São Paulo; e o curso de Psicologia Social e Econômica, ministrado por Nilton Campos, na década de 1950, na Faculdade Nacional de Ciências Econômicas da Universidade do Brasil.

HISTÓRICOS CURSOS DE

PSICOLOGIA SOCIAL NO BRASIL

O pioneiro curso de Psicologia Social ministrado por Raul Briquet na Escola Livre de Sociologia e Política de São Paulo resultou na publicação do seu livro Psicologia Social (1935). Nessa época, Raul Briquet já formava, juntamente com Roberto Simonsen, Jorge Street, Armando de Sales Oliveira, Pacheco e Silva e André Dreyfuss, a lista dos mantenedores da Escola Livre de Sociologia e Política de São Paulo, que acabou sendo anexada à Universidade de São Paulo em 6 de dezembro de 1938.

Raul Briquet, um paulista nascido em Li- 
meira no ano de 1887, era filho de um engenheiro francês interessado em trabalhar nas estradas ferroviárias que se abriam no Brasil. Após conhecer a filha de um diplomada brasileiro em missão na França, casou-se e constituiu família, que cresceu nos caminhos de uma estrada ferroviária que se construía no interior do Estado de Minas Gerais. Graças à requintada formação de sua mãe, sua única professora, com quem adquiriu conhecimentos de idiomas e de música, conseguiu ingressar na Faculdade de Medicina do Rio de Janeiro, onde defendeu sua tese relativa à psicofisiologia e à patologia musical. Era poliglota, exímio pianista, conhecido como sendo um devorador de livros e um ser humano muito solidário, conforme relata o livro Raul Carlos Briquet (BOMFIM, 2002).

Sua especialização em ginecologia e obstetrícia e suas publicações na área, a partir de 1914 propiciaram a criação de uma postura científica, conhecida como "Escola Briquet de Ginecologia". Em 1925, assumiu a cátedra de "Clínica Obstétrica" da Faculdade de Medicina e Cirurgia de São Paulo. Em 1927, juntamente com Franco da Rocha, Durval Marcondes e Lourenço Filho, participou da criação da "Sociedade Brasileira de Psicanálise", primeira entidade associativa dos psicanalistas brasileiros, tendo ocupado o cargo de vicepresidente. Em 1930, envolveu-se na criação da "Sociedade de Filosofia e Letras de São Paulo", instituição que acabou viabilizando a fundação da Universidade de São Paulo, onde Briquet tornariase Catedrático de Clínica Obstetrícia e Puericultura Neonatal. Briquet foi, também, signatário de um dos mais importantes documentos da educação brasileira: o "Manifesto dos Pioneiros da Escola Nova", ou mais comumente conhecido como "Manifesto dos Pioneiros", publicado em 1932

Foi nessa condição que Briquet propôs, em 1933, o curso de Psicologia Social à Escola Livre de Sociologia e Política. O primeiro contato com o material legado por Briquet revela que, no seu curso, uma vasta e atualizada lista de referências bibliográficas foi facultada aos alunos.

Iniciando sua performance com uma apresentação das principais contribuições à Psicologia Social advindas da Biologia, da Psicologia e da Sociologia, Briquet deu a cada uma dessas contribuições um peso semelhante. Num tratamento igualitário, posicionou a Psicologia Social como um campo dependente tanto da Psicologia quanto da Sociologia e da Biologia. Nessa parte do curso, considerada como parte geral, articulavam-se uma introdução, uma apresentação dos subsídios da Biologia, uma apresentação dos subsídios da Psicologia - incluindo o behaviorismo, o gestaltismo, as
Psicologia \& Sociedade; 16 (2): 32-36; maio/ago.2004 leis da natureza humana e a aprendizagem - e uma apresentação dos subsídios da Sociologia.

Na segunda parte, denominada parte especial, a relevância era direcionada aos fatores psíquicos e à vida social, situando, reflexivamente, as seguintes temáticas:

Fatores psíquicos: instinto

instinto agressivo

hábito

sugestão

imitação

simpatia

inteligênciea

Vida social:

grupos sociais

eu social

personalidade adaptação socia preconceito de raça liderança opinião pública multidão

revolução

Ao engendrar-se nas temáticas específicas da Psicologia Social, Briquet privilegiou, em primeira instância, os fatores psíquicos que motivavam o comportamento social, relacionando o instinto, o hábito, a inteligência e o que ele considerava como as três formas de identidade social: a sugestão, a imitação e a simpatia. Num segundo momento, Briquet articulou, em sua concepção de vida social, os grupos sociais, o eu social, a personalidade, a adaptação social e - o que nomeou como Psicologia Coletiva - as questões relativas ao preconceito de raça, à liderança, à opinião pública, à multidão e à revolução.

Quanto aos métodos e medidas, Briquet, ampliando a questão além da experimentação, relevava quanto o método experimental seria aleatório em Psicologia Social devendo, dessa maneira, harmonizar-se com outros métodos. Ele propunha a utilização de análise de biografias, autobiografias e casuística, além de alguns métodos especiais para o estudo do eu social, como a medida de distância social proposta por Bogardus.

Seu estudo sobre grupos foi fortemente influenciado pelo materialismo histórico de Karl Marx (abrangendo o determinismo econômico, a concepção materialista da história, a luta de classes e a teoria econômica) e pelo método dialético de Hegel. Com isso, concorria Raul Briquet para a introdução da visão materialista histórica em Psicologia Social no Brasil, conforme Bomfim (2003).

Sua atitude anti-racista levou-o a um ferrenho combate ao preconceito racial num momento histórico em que - aos preconceitos contra negros e mulatos - eram acrescidas as barreiras à imigração japonesa. Posicionou-se, ainda, contra a censura e defendeu a possibilidade de revolução contra um governo injusto. 
Bomfim; E.M. "Históricos Cursos de Psicologia Social no Brasil"

Privilegiou autores como Dewey, James, Hall, Watson, Thorndike, Politzer, Woodworth, Bechterew, Pavlov, Koffka, Hohler, Wertheimer, Ribot, Sorokin, Comte, Delacroix, Condorcet, Freud, Jonas, Wundt, Simmel, Tarde, Durkhleim Blondel, Essetier, Duprat e Klineberg e relacionou os, então recentes, manuais de Psicologia Social: de Ellwood, de Allport, de Murphy e Murphy, de Murchison, de Folsom, de Bernard, de Kruger e Reklless, de Young e de Gault, dentre outros. Mencionou, também, as revisões históricas de Psicologia Social feitas por Geck, na Alemanha, e por Karpf, nos Estados Unidos. Entre os brasileiros, Briquet privilegiou, entre outros, Oliveira Vianna, Salles, Lins, Medeiros e Albuquerque, Kehl, Neves-Manta, Nina Rodrigues, Porto-Carrero, Roquette-Pinto, Miguel Couto e Arthur Ramos, em sua já publicada obra sobre o negro brasileiro.

Foi, exatamente, Arthur Ramos o responsável pelo curso de Psicologia Social ministrado, entre julho e dezembro de 1935, na Escola de Economia e Direito da extinta Universidade do Distrito Federal situada no Rio de Janeiro. Do curso, resultou a publicação do livro Introdução à Psychologia Social (1936).

Na ocasião, Arthur Ramos de Araújo Pereira (1903-1949) já era conhecido por suas publicações, ressaltando entre elas: Estudos de Psicanálise (1931), Psiquiatria e Psicanálise (1934), O negro brasileiro (1934), Educação e Psicanálise (1934) e $O$ folk-lore negro do Brasil (1935). Assim como Raul Briquet, Artur Ramos teve sua graduação em Medicina, tendo estudado na Bahia e tido por mestre Nina Rodrigues, que o orientou em suas primeiras pesquisas.

Pelos desdobramentos de seu curso, é possível constatar similaridade, em vários tópicos, ao curso de Briquet, cuja obra foi objeto de referência. Além de Briquet, Ramos apoiava-se em autores como Thorndike, Watson, Blonsky, Blondel, Dewey, Tarde, Vigotski, Luria, Pavlov, Koffka, Kohler, Aron, Bion, Kornilov, Freud, Adler, Jung, Reich, Ferenczi, Gesell, Adam Smith, Piaget, McDougall, Ross, Bogardus, Gault, F. Allport, Ellwood, Bernard, Murphy e Murphy, Young e Murchison. Dos brasileiros, Ramos privilegiou, entre outros, Juliano Moreira, Afrânio Peixoto, Porto-Carrero, Fernando de Azevedo, Delgado de Carvalho, Gustavo Lessa, Lourenço Filho, Anísio Teixeira, Sílvio Romero e o mestre Nina Rodrigues. Para Ramos, a Psicologia Social, uma disciplina entre a Psicologia e a Sociologia, estava em crescente importância, embora não tivesse seus métodos e objetivos ainda claros. Resultava daí a imprecisão em sua própria nomeação, sendo deno- minada como interpsicologia, psicologia social, psicologia coletiva, psicologia das raças, psicologia dos povos, psicologia das multidões ou psicologia das seitas. O próprio Ramos nomeava o profissional da área ora como psicossociólogo, ora como sociopsicólogo. Ainda que imprecisa em sua definição, à Psicologia Social caberia o estudo das bases psicológicas do comportamento social, das inter-relações psicológicas dos indivíduos na vida social e da influência dos grupos sobre a personalidade.

Tal como Briquet, Ramos forneceu um panorama geral da Psicologia, tendo acentuado as contribuições do behaviorismo, da psicanálise e do gestaltismo. Tratou de forma semelhante temas como a sugestão, a imitação, a simpatia, a opinião pública, a censura e a propaganda. Adentrou nos fundamentos da Psicologia Social, na biologia dos motivos, no hábito, na aprendizagem social, nas estruturas instintivo-afetivas, nas reações da personalidade, na interação mental, na interferência, no conflito e nos desajustamentos psicossociais. Abordou, ainda, a vida dos grupos, a relação entre o individual e o social, a psicologia da cultura, a estrutura da mentalidade primitiva, a lógica afetiva e sua relação com o pensamento mágico-simbólico, as esferas primitivas da realidade e a sobrevivência das estruturas primitivas.

Uma importante contribuição do curso de Ramos foi a articulação da Psicologia Social com a Antropologia Social, fundamentada nos escritos de Malinowski, Franz, Boas e Lévy-Bruhl. Numa perspectiva culturalista, Ramos propunha uma nova ordenação que seria a Psicologia Social Comparada que, ao complementar a Antropologia Cultural, facultaria, pelo seu ponto de vista cultural, a correção do critério evolucionista linear, permitindo a evolução psicológica dentro de suas culturas.

Metodologicamente, Ramos propunha a utilização dos métodos fisiológicos e morfológicos, dos métodos biográficos, dos métodos de "impressão pessoal", dos métodos de questionários e entrevistas e dos métodos de testes.

Entre os vários pontos de congruência nos dois cursos, ressalta a visão panorâmica da Psicologia Social presente tanto no curso de Ramos como no de Briquet.

Essa abordagem abrangente seria contrastada, na década de 1940, num terceiro curso, cujo legado documental foi possível acessar. Trata-se do curso ministrado por Donald Pierson na Escola Livre de Sociologia e Política da Universidade de São Paulo. O referido curso foi registrado no livro Teoria e Pesquisa em Sociologia (1945), uma coletânea de artigos sobre Sociologia e Ecologia Hu- 
mana.

Donald Pierson, quando o publicou em 1945, já estava há seis anos no Brasil lecionando Sociologia, Psicologia Social e Antropologia Social na Escola Livre de Sociologia e Política de São Paulo. Havia organizado o Departamento de Sociologia e Antropologia Social, transformado, em 1943 em Divisão de Estudos Pós-Graduados. Entre seus trabalhos técnicos, já destacava-se a coordenação da coleção "Biblioteca de Ciências Sociais" e a publicação da obra Negroes in Brazil: a study of race contact at Bahia (1942). Posteriormente, organizaria as publicações de Estudos de Ecologia Humana (1948) e de Estudos de Organização Social (1949)

Tendo sido orientando de Robert Park (1864-1944), o fundador da "Escola de Chicago", Pierson trazia em sua formação a influência de seus professores de Chicago, entre eles: George Mead. Com tal bagagem, iria registrar, como objetivos do seu curso de Psicologia Social, o estudo dos meios pelos quais o organismo homo sapiens assumiria a natureza humana e, ao mesmo tempo, a análise de como a pessoa resultante desse processo se in corporaria ao seu grupo social, através da aquisição de um self que a capacitaria ao controle pessoal e social e à participação de uma ação conjugada. A influência da obra de Mead e o espaço privilegiado dado à abordagem do interacionismo social não o impediram, contudo, de dar uma visão abrangente da origem da Psicologia Social, da classificação dos psicólogos sociais e da relação entre a Psicologia Social e as ciências sociais. Entre as concepcões e os conceitos fundamentais da Psicologia Social Sistemática, incluiu a noção do indivíduo, os conceitos de objeto, significado, comportamento, conduta, gestos, sentimentos, bloqueio, desejos, frustração, sublimação, perversão, racionalização, neurose, rapport, imitação, sugestão, simbolismo, self, comunicação e a categoria "do assumir o papel de outrem".

Pierson entendia, ainda, a Psicologia Social como um subcampo tanto da Sociologia quanto da Psicologia.

Sua vasta e atualizada lista de referências bibliográficas incluía, como proposta de leitura in dispensável, as obras de George Mead - sua principal referência bibliográfica -, Dewey, Cooley, G. Allport, Thomas, Young, Adler, Sullivan, Koffka e Karpf. Como leitura suplementar, propunha uma relação de cerca de 50 livros que influíram na formação da Psicologia Social, incluindo os autores Wundt, Durkheim, Berkeley, Hobbes, Hume, Simmel, Spencer, Bekhterev, Lazarus, Stern, Pavlov, Franz Boas, Stanley Hall, Judd, Ross, Le Bon, Levy Bruhl, Freud, Jung e Bernard. Relacionou, ainda, uma lista de estudos selecionados, composta por textos de Park, Burgess, Faris, Smith e Wright, Thurstone, Znaniecki, Kardiner, Kluckhohn, Lewin, F. Allport, Kohler, Margaret Mead, Murphy, John Dollard e Stern. E, finalmente, numa seção denominada de esforços para resumir ou sistematizar o campo, incluiu os textos de Raul Briquet, Arthur Ramos, Otto Klineberg, Bernard, Ellwood, Folson, Gault, Gordon, Murchison, Reinhardt, Znaniecki e W. McDougall.

À ampla lista de referências bibliográficas contrasta-se a ausência de referência no registro legado por Nilton Campos, um dos introdutores do pensamento fenomenológico no Brasil, que, na década de 1950, foi o responsável pelo Curso de Psicologia Social e Econômica, da Faculdade Nacional de Ciências Econômicas, instituição vinculada à antiga Universidade do Brasil situada no Rio de Janeiro.

O carioca Nilton Campos (1898-1963), formado na Faculdade Nacional de Medicina (1923), já havia defendido, em 1945, a tese "O método fenomenológico na Psicologia", para o provimento da Cátedra de Psicologia na Faculdade Nacional de Filosofia da antiga Universidade do Brasil e, em 1948, assumido a direção do "Instituto de Psicologia" em substituição à Jaime Grabois. Trazia em sua bagagem profissional a publicação de seu livro Psychologia da vida affectiva (1930) e a experiência na Colônia de Psicopatas do Engenho de Dentro, onde foi assistente de Waclaw Radecki e trabalhou no Laboratório de Psicologia da Colônia de Psicopatas Engenho de Dentro com companheiros como Helina Radecka, Lucília Tavares, Ubirajara da Rocha, Arauld Bretas, Alberto Moore, A. Bulhões Pedreira e Euryalo Cannabrava.

No Instituto de Psicologia, Nilton Campos criou o Boletim do Instituto de Psicologia, em 1951, no qual publicava periodicamente. Foi nesse periódico que Campos divulgou, em 1952, o programa do seu curso de Psicologia Social e Econômica.

O curso de Psicologia Social e Econômica de Nilton Campos continha 27 tópicos sobre diversas temáticas que incluíam: a) conceito e relações da Psicologia Social com as ciências afins; b) objeto da Psicologia Social; c) natureza dos fenômenos psicossociais; d) fases do desenvolvimento da Psicologia Social; e) métodos de pesquisa; f) natureza das relações entre o indivíduo e a sociedade; g) fenômeno de interação; h) isolamento social; i) identidade humana e diversidade de culturas; j) percepção e cognição; k) cultura e personalidade; 1) formação e integração da personalidade; m) impulsos, desejos, instintos e tendências; n) crenças 
Bomfim; E.M. "Históricos Cursos de Psicologia Social no Brasil" e atitudes; o) grupo social; p) relações inter-humanas; r) tensões sociais; s) criminalidade e infância abandonada; t) sugestibilidade, imitação, simpatia e antipatia; u) propaganda e opinião pública; v) liderança; x) organização científica do trabalho e desajustamento no trabalho; w) costumes e moda; y) progresso nas investigações científicas da psicologia; e z) apreciação das fontes bibliográficas.

Na questão metodológica, Nilton Campos propunha a utilização de métodos de pesquisa vinculados às correntes behaviorista, gestaltista e psicanalítica e nomeava as possibilidades e as limitações da pesquisa experimental.

A ausência - quer dos objetivos, quer das fontes bibliográficas - deixou, contudo, lacunas irremediáveis, limitando a análise à uma exposição de temáticas.

Finalmente, vale ressaltar que, situando reflexivamente, todos os cursos estiveram estreitamente vinculados à construção do pensamento em Psicologia Social no Brasil. Eles apontam que, até meados do século $\mathrm{XX}$, a prioridade na busca do status de conhecimento científico para o campo psicossocial realizava-se num clima de alegada falta de espírito universitário de pesquisa e da ausência de objetividade e de imparcialidade necessárias ao país.

À visão generalista, própria da década de 1930, buscou-se a alternância através do privilégio a determinadas abordagens teóricas nas décadas seguintes. Contudo, pelos seus desdobramentos basilares, observa-se que persistiram os dois modelos na segunda metade do século XX. São contribuições históricas, importantes marcos no passado, que deixam, para os interessados, registros relevantes sobre a Psicologia Social no Brasil.

\section{REFERÊNCIAS:}

BOMFIM, E. Raul Carlos Briquet. Rio de Janeiro: Imago, 2002.

BOMFIM, E. Psicologia Social no Brasil. Belo Horizonte: Edições do Campo Social, 2003.

BRIQUET, R. Psicologia Social. São Paulo: F. Alves, 1935.

CAMPOS, N. Psychologia da vida affectiva. [Rio de Janeiro: s. n.], 1930.

CAMPOS, N. O método fenomenológico na Psicologia. 1945. Tese (Doutorado) - Faculdade Nacional de Filosofia, Rio de Janeiro, 1945.
CAMPOS, N. Programa de Psicologia Social e Econômica da F.N.C. Econômicas. Boletim do Instituto de Psicologia, Rio de Janeiro, 21(3-4), 21-23: 1952.

PIERSON, D. Negroes in Brazil: a study of race contact at Bahia. Chicago: University of Chicago, 1942. (Brancos e Pretos na Bahia. São Paulo: Ed. Nacional, 1944.).

PIERSON, D. (Org.). Estudos de Ecologia Humana. São Paulo: Martins, 1948.

PIERSON, D. (Org.). Estudos de Organização Social. São Paulo: Martins, 1949.

PIERSON, D. [1945] Teoria e Pesquisa em Sociologia. 12. ed. São Paulo: Melhoramentos, 1970.

RAMOS, A. Estudos de Psicanálise. [Salvador: s. n.], 1931.

RAMOS, A. Educação e Psicanálise. [São Paulo: s. n.], 1934

RAMOS, A. O negro brasileiro. Rio de Janeiro: Civilização Brasileira, 1934.

RAMOS, A. Psiquiatria e Psicanálise. [Rio de Janeiro: s. n.], 1934.

RAMOS, A. O folk-lore negro do Brasil. Rio de Janeiro: Casa do Estudante, 1935.

RAMOS, A. Introdução à Psychologia Social. Rio de Janeiro: J. Olympio, 1936. (2. ed. Rio de Janeiro: Casa do Estudante do Brasil, 1952.).

Elizabeth de Melo Bomfim é pesquisadora visitante no Laboratório de Pesquisa e Intervenção Psicossocial da Universidade Federal de São João Del-Rei, com bolsa da Fundação de Amparo à Pesquisa do Estado de Minas Gerais. Foi presidente da Associação Nacional de Pesquisa e Pós-Graduação em Psicologia - ANPPEP (gestão 1994-1996), presidente da Associação Brasileira de Psicologia Social - ABRAPSO (gestão 1987-1989 e gestão 1997-1998) e Coordenadora do Programa de Pós-Graduação em Psicologia da UFMG (gestão 1991-1994). O endereço eletrônico da autora é: embomfim@brfree.com.br

Elizabeth de Melo Bomfim

Históricos cursos de

psicologia social no Brasil

Recebido: 4/3/2004

$1^{\text {a }}$ revisão: 6/5/2004

Aceite final: $21 / 6 / 2004$ 